\title{
Entrevista a Alfonso Dávila Oliveda: Archivero del Cuerpo Facultativo de Archivos
}

\author{
ENTREVISTA REALIZADA POR ANA NASEIRO RAMUDO
}

Vocal de SEDIC

\begin{abstract}
Entrevista concedida por D. Alfonso Dávila Oliveda, destacado archivero y gran profesional de los Archivos Estatales españoles, quien ha ejercido su labor profesional de manera continuada en los archivos históricos. D. Alfonso Dávila Oliveda muestra en esta entrevista su experiencia humana y profesional en la dirección de dos destacados archivos, asimismo, nos invita a conocer su faceta como escritor biógrafo-historiador de Miguel Cervantes de Saavedra.
\end{abstract}

Archivero, Historiador, Miguel Cervantes de Saavedra, Archivo General de la Administración, Archivo Histórico Provincial de Álava.

\section{Introducción}

Alfonso Dávila Oliveda, es un gran profesional de los archivos, yo he tenido la suerte de trabajar bajo su Dirección en el Archivo General de la Administración (AGA) durante ocho años y ahora se me ha presentado la oportunidad de entrevistarle. En mis años de estancia en el AGA, Alfonso era una persona seria y discreta, que nunca ha buscado llenar su ego con un reconocimiento no merecido, aunque en mi opinión es un maestro de la archivística, un trabajador incansable que ha tratado de convertir el mastodóntico y sin recursos AGA en un referente y de transformar la archivística en un modelo de innovación en la transformación digital y administrativa. Alfonso tiene una gran capacidad de reflexión y creación que se ha manifestado y manifiesta en múltiples conferencias y exposiciones que generaba sin dificultad y con gran ilusión.

Me gustaría destacar su gran calidad como historiador que se ha plasmado en las publicaciones inéditas publicadas en los últimos años en los que ha se ha centrado en el estudio del insigne escritor Miguel de Cervantes y Saavedra, dando a la luz importantes descubrimientos de repercusión internacional en torno a su biografía que nos acercan más al lado humano de dicho personaje y su época, sus investigaciones sobre el genio de la literatura española publicadas hasta el momento son: "Miguel de Cervantes. Apuntes para una biografía. Vol. Soldado poeta”; “Miguel de Cervantes. Apuntes para una biografía. Vol. II. El agente del rey, predestinado para el teatro que se dedicaba a los negocios (1586-1595)". "Miguel de cervantes. Apuntes para una biografía. Vol III. El espía. (1595-1603)”. “El espía de Felipe II que acabó con Hasan Baja, virrey de Trípoli y Argel (1595-1601). Apuntes para una biografía III”. Y que en breve esperamos que publique el volumen IV. También resulta de gran interés su "Guía Itinerario de Miguel Cervantes y Alcalá de Henares", que permite realizar un recorrido por la vida del escritor en la ciudad complutense. 
Recomiendo encarecidamente la lectura de todas estas obras, ya que Alfonso ha rescatado en ellas al Cervantes poeta y dramaturgo, además de novelista, sus oficios fuera del ámbito de las letras: comerciante, soldado, espía, recaudador de impuestos, dramaturgo, entre otros, así como sus relaciones intrigantes con grandes linajes e importantes familias y personajes coetáneos, como la familia Mendoza o figuras como Lope de Vega, entre otras familias y poetas y dramaturgos de la época. Otros aspectos que nos ha acercado Alfonso del insigne escritor ha sido su amplio recorrido geográfico, las andanzas del Cervantes aventurero, serán el origen de la inspiración de sus grandes novelas. Alfonso nos ha sorprendido con otros descubrimientos como las relaciones entre la Universidad de Alcalá y México y el patrocinio de los Cervantes en esta última. Dichas revelaciones basadas en un estrecho contacto y dominio en la búsqueda en los archivos y no únicamente en referencias bibliográficas o fuentes citadas, como se ha reflejado en otras biografías del autor, muestran su gran dominio de las fuentes documentales y de la investigación histórica.

\section{Cuestionario}

1. La gran mayoría de su carrera profesional la ha ejercido en el Archivo General de la Administración, ha estado en dos etapas muy diferentes en la primera como técnico de archivos y en la segunda como Director.

¿Qué diferencias ha observado en la institución en esas dos etapas?

Etapa de 1986 a 1996:

Antes de hablar de dos etapas diferenciadas, tengo que señalar que ingresé en el Archivo General de la Administración, tras superar un concurso oposición de personal laboral fijo de técnico de archivos para el Archivo General de la Administración Civil del Estado (AGA) con la categoría laboral de titulado superior nivel 1, proceso selectivo que fue un intento por parte de la Administración General de igualar el acceso del personal de las Administración Pública Española a las formas de ingresos de los países que entonces conformaban la Comunidad Europea. La oposición la gané tras superar un proceso selectivo consistente en primer lugar en un test de evaluación sicológica, una vez superado el mismo, tuve que hacer frente a un examen por escrito de tres temas sacados al azar, de historia, archivística y legislación administrativa, proceso que se desarrolló durante un periodo de 6 horas, una vez leídos los temas ante el tribunal y obtenida la calificación tuve que desarrollar un examen de idioma moderno y superado el mismo realizar el examen de un supuesto práctico archivístico, que consistió en la ordenación y descripción de un expediente del siglo XIX complemente desorganizado, que igualmente tuve que exponer y defender ante el tribunal.

Cuando ingresé en esta etapa, yo conocía profundamente el AGA, ya que mi promoción de archiveros de la Escuela de Biblioteconomía y Documentación de la Biblioteca Nacional de 1981/1982, pudo dedicar una parte de su formación en la escuela ,en jornada de tarde, a dedicar quince días de prácticas en horario de mañana, en los archivos de Madrid, Histórico Nacional, Banco de España, Protocolos Notariales, el AGA y algún ministerio, no sé por qué me decidí por el AGA, donde tuve la suerte de ser formado por Ignacio Ruiz Alcain, Técnico Superior de 
Archivos, personal laboral fijo, Nivel 1, Jefe de Sección de los fondos documentales del Ministerio de Asuntos Exteriores, por lo que en vez de permanecer quince días conseguí prolongar mis prácticas durante todo el año académico de post licenciatura, antecedente de los actuales máster, hasta la entrega de mi Diploma de Archivero en el mes de junio de 1982.

Cuando volví en 1986, después de haber trabajado como Archivero, como personal laboral temporal Nivel 1, en el Archivo Histórico Provincial de Burgos, contratos en los que tenía que superar un examen de evaluación sicológica, que realizaba el Instituto Nacional de Empleo a los demandantes de trabajo. Participé en el Censo Guía de Archivos de la provincia de Burgos, contratado por el Centro de Información Documental de Archivos (CIDA), dependiente de la Subdirección General de Archivos y de la Diputación Provincial de Burgos, y en la organización y descripción de los Archivos Municipales, tras ganar una beca de la Diputación Provincial de Burgos, precisamente aprobé las oposiciones a mi puesto laboral fijo en el AGA, cuando estaba inventariando el Archivo Municipal de Espinosa de los Monteros y su Fondo Documental de los Monteros de Espinosa, que era la guarda personal de los Reyes de España hasta la proclamación de la Segunda República Española.

En febrero de 1986 María Luisa Conde Villaverde, profesora de la Escuela de Biblioteconomía y Documentación, acababa de asumir la dirección del AGA, tras su paso por el Ministerio de Comercio y la implantación de su proyecto archivístico de informatización, denominado SCAA, con el fin de adaptar la organización de los Archivos Administrativos a las nuevas tecnologías, lo que hoy denominamos Archivos de Gestión Electrónicos, que en aquellos años eran sólo archivos tradicionales en papel en los que se implantaban las nuevas tecnologías informáticas a la clasificación y descripción documental, pero no a la sustitución de sus soportes por soportes electrónicos y digitales, como en la actualidad. No es lo mismo el soporte electrónico que es el documento realizado íntegramente en cadenas de bit electrónicos o cuánticos, que la administración del documento digital, que es la gestión de la información de los soportes documentales digitales de audio, imagen fija o en movimiento.

Entre 1986 a 1996, diferencio tres fases en mi paso por el AGA:

La primera de apoyo a las secciones del Archivo, Sindicatos, Información y Turismo, Cultura, Educación, Obras Públicas, basada fundamentalmente en la atención a los investigadores en la Sala de Consulta y en el apoyo a secciones archivísticas con grandes volúmenes documentales desorganizados, pero vitales para la gestión de la administración y de los ciudadanos, en este sentido la sección de Sindicatos era vital para atender las jubilaciones y derechos de los ciudadanos, ya que era el organismo que había gestionado los fondos de pensiones y de mutualidades laborales. En esta etapa elaboro mi Guía de Fuentes para la Historia de América.

La segunda, entre 1992 y 1994, asumo la responsabilidad de la gestión de lo que se conoce como Sección de Asuntos Exteriores, etapa que compagino con la formación de los alumnos en prácticas archivísticas de las Escuelas Taller de Archivos y la implantación del SCAA en el propio AGA, consistente en la identificación de las Series Documentales y la implantación de las bases de datos electrónicas y la normalización del control topográfico de los depósitos documentales en bases de datos, cuyo objetivo era implantar el Sistema de gestión y difusión electrónica y digital del proyecto del Archivo General de Indias. En esta etapa me dedico con grupos de trabajo de las escuelas taller a la organización de grandes volúmenes documentales desorganizados, además son los inicios de los trabajos de Valoración de Fondos Documentales para su conservación permanente o eliminación, participando en los Grupos de Trabajo de 
Valoración, de la Subdirección General de Archivos Estatales de Hacienda, Justicia, Sindicatos y Gobiernos Civiles fundamentalmente. Participo plenamente, como profesor, en los Cursos de Formación Archivística del INAP o de los distintos ministerios u Organismos Autónomos, como la formación del personal del recién creado Instituto Cervantes.

La tercera, la más corta, pero la más intensa, centrada exclusivamente en los trabajos de Valoración para el acceso a la documentación y sobre todo para la preservación o eliminación de los grandes volúmenes documentales, trabajos en los que se integran la formación y la colaboración de los alumnos de las escuelas taller de archivos, junto a la contratación de técnicos especializados en los trabajos de valoración, que se habían formado en los años anteriores en el propio AGA. Etapa que acaba con la obtención de plaza del Cuerpo Facultativo de Archiveros, Bibliotecarios y Arqueólogos en el concurso oposición de funcionarización del personal laboral, concurso oposición agridulce, ya que suponía el fracaso de la adaptación del acceso del personal de la administración pública al modelo de acceso de la comunidad europea, fracaso que incluso se vivió en el cambio radical de la gestión archivística en el AGA, ya que se ralentizaron los estudios de valoración, lo que impidió desarrollar la Comisión Superior Calificadora de Documentos Administrativos, a efectos de impacto externo, porque ya internamente en la organización del trabajo del AGA se había producido una revolución de la gestión archivística y ya no hubo marcha atrás. El SCAA, no se implantó, pero los cambios habían conseguido organizar sus grandes volúmenes documentales, normalizar por medios electrónicos los depósitos documentales y establecer las bases de datos de acceso a los fondos documentales más demandados por los ciudadanos y la administración, consiguiendo una buena ratio de efectividad entre el tiempo empleado en la localización y transmisión de la información, lo que significó que la información o el documento original o su copia podía encontrarse en la oficina que lo demandaba en el mismo día.

Etapa de 2006 a 2015:

La segunda etapa, fue la que más me costó aceptar, ya que llevaba 10 años viviendo en Vitoria, donde mis hijos habían crecido y formado sus cuadrillas de amigos y había conseguido integrar el Archivo Histórico Provincial de Álava en la propia esencia ciudadana. Con el apoyo del Ministerio de Cultura, de la Delegación y Subdelegación de Gobierno y la aprobación del Gobierno Vasco y de la Diputación Foral de Álava se había dotado de un edificio de nueva planta, que garantizaba la conservación y restauración documental de sus fondos, la informatización de las descripciones documentales, de las relaciones de entrega de las transferencias documentales. Archivo que se había integrado en los sistemas de archivos del Gobierno Vasco y de la Diputación Foral de Álava, colaborando estrechamente con los archivos municipales y las asociaciones de archiveros, así como con las fundaciones y organismos colegiados de la provincia. Colaboraba estrechamente en el plan de formación archivístico del Gobierno Vasco a través de los cursos de archivos administrativos organizados por el IVAP, colaboraba estrechamente en los planes de formación en archivos administrativos de las diputaciones forales de Vizcaya y Álava y coordinaba el Máster de Archivos, que la Universidad del País vasco desarrollaba en el campus de Álava, en su Facultad de Historia.

Analicé entonces con mi familia, la propuesta de la Dirección del AGA, realizada por el Subdirector General de los Archivos Estatales, amigo y excelente compañero, en los cursos de formación de los archivos administrativos de las instituciones archivísticas autonómicas y forales, al que solo le puse una condición para asumir la Dirección del Archivo General de la 
Administración, que debía nombrarse un Director del Archivo Histórico Provincial de Álava, antes de mi aceptación de la dirección del AGA, lo que se llevó a cabo y yo asumí en adscripción provisional la dirección del archivo en abril del año 2006.

Sustituí entonces a mi maestra, profesora, directora y siempre amiga María Luisa Conde Villaverde, que había sido cesada unos meses antes en la dirección del AGA en una situación administrativa y política complicada, tiempos difíciles, que había provocado que distintos Facultativos del Cuerpo de Archivos, renunciasen a sus nombramientos tanto a la Subdirección de Archivos Estales como a la Dirección del AGA, cuando sus nombramientos se encontraban pendientes de publicación en el Boletín Oficial del Estado.

Aún recuerdo el primer día que me presenté como director provisional del AGA, a las 7,30 horas de la mañana, recibiendo y presentándome a cada uno de los empleados públicos del archivo. En ese momento tomé una decisión, no tenía amigos ni conocidos en el archivo, que todo el personal estaba altamente cualificado, que trabajaban eficazmente pero que, debido a la distancia de la sede central del Ministerio, nunca había sido valorado como se merecía. La verdad, es que la franca y estrecha colaboración que recibí del personal del archivo, contribuyeron a establecer una nueva planificación y reorientación del sentido del Archivo Intermedio, en los inicios de lo que denominamos Era Digital.

Apliqué los principios archivísticos que me enseñaron los archiveros que me precedieron en el puesto y sobre todo la enseñanza y formación que recibí de mis profesores en la Escuela de Biblioteconomía y Documentación, cuyas enseñanzas se habían orientado a la planificación de nuestra especialidad a lo que se preveía como nueva revolución electrónica, cuya implantación ocasionaría, como en la revolución industrial del siglo XIX, la readaptación de las tecnologías del trabajo a los nuevos instrumentos y planificaciones que implicaban la investigación y la aplicación de las nuevas tecnologías.

Para ello, planifiqué los cambios que se debían producir, tanto en las infraestructuras como en la organización del personal.

Sin duda la parte más sencilla fue la adaptación de las infraestructuras del edificio o continente de conservación de la documentación, con la reorientación de las obras de ampliación, que se estaba ejecutando a mi llegada de acuerdo con la previsión de las nuevas necesidades que requerían las nuevas tecnologías que se estaban implantado a nivel mundial y con un estudio de las mejoras necesarias para la adecuación de los depósitos y salas de trabajo a las nuevas necesidades tecnológicas, lo que originó una segunda fase de obras, que remodeló por entero el edificio administrativo sin que la obras repercutiese en los investigadores, en el trabajo diario o en los servicios a la administración, a los ciudadanos y a los investigadores.

Uno de los primeros cambios fue la implantación de la digitalización de la documentación en detrimento de la microfilmación. Es curioso como los técnicos del Archivo se adaptaron y defendieron la implantación de las nuevas tecnologías digitales, convirtiéndose en pioneros en sus especialidades, elaborando y diseñando programas informáticos de conservación y difusión, que cristalizaron y permitieron la incorporación del AGA en los grandes proyectos estatales y comunitarios como PARES, la Biblioteca Digital Hispana, y la implantación en el propio Archivo de ANDREA, que permite que todos los usuarios del centro, incluidos los investigadores puedan acceder a todo tipo de soporte de descripción de la documentación, que no se encuentre en modelos normalizados electrónicamente. Hoy es un instrumento de acceso imprescindible en un centro de las características del archivo intermedio de la Administración General del Estado.

La implantación digital implicó hacer frente a las tensiones que se produjeron por el fin de la microfilmación, definida como "Memoria de la Humanidad" por la UNESCO ya que la "Era 
Digital” es en sí misma la Memoria Global Planetaria, sin limitaciones de ámbitos geográficos o políticos.

La parte más complicada y la que está pendiente de realización, es la adaptación de la estructura de la plantilla de personal a las nuevas categorías y especialidades laborales que requiere una institución como el Archivo Intermedio y su adecuación e integración en el Archivo Único Electrónico de la Administración General del Estado.

Con este fin se desarrolló una propuesta de modificación del organigrama del personal, con adaptación de las denominaciones de los puestos a las nuevas categorías profesionales y administrativas, necesaria para la implantación de las nuevas tecnologías y especialidades, que deben abarcar desde la fotogrametría, robótica, preservación y restauración de los soportes de memorias electrónicos y digitales, laboratorios de investigación para la migración y preservación de datos y documentos, adaptación a la profesión archivística de ingenieros electrónicos y digitales, etc. Es curioso observar como las propuestas de entonces empiezan a cuajar en las altas esferas de la Administración, por lo que pienso que en una década veremos cómo las planificaciones empezarán a ser realidad en las plantillas de personal de los archivos administrativos e históricos.

¿Qué le une a dicha institución para desear volver a ejercer en la misma?

Sinceramente, yo hubiese sido feliz retornado a mi ciudad de nacimiento, a mi querido y malogrado archivo histórico provincial de Burgos, tan maltratado por los políticos de miras estrechas y unas instituciones municipales, provinciales y autonómicas carentes de sensibilidad ante el patrimonio documental del nacimiento del condado de Castilla y del castellano, base hoy del español. Espero que algún día sea reconocido y consiga tener las instalaciones que se merece, porque la penosa imagen que da este archivo histórico provincial es la punta de lanza que se observa en la provincia burgalesa, ante la dejación en la preservación de su patrimonio histórico artístico.

A pesar de mis sentimientos, me incliné por el AGA, como un reto personal para desarrollar las teorías archivísticas, que me inculcaron y la adaptación a las nuevas tecnologías, sin mirar el color administrativo del acceso a la función pública. La verdad es que cuando asumí mi puesto de trabajo como personal laboral fijo de técnico superior de archivos del Archivo General de la Administración en 1986, comprendí lo que podía sentir una persona discriminada por sus creencias, deficiencias físicas o el color de su piel o adscripción administrativa laboral o funcionario.

Siempre agradeceré la profesionalidad y amplitud de miras de tres personas que fueron Subdirectores Generales de Archivos en mi carrera profesional, Luis Mata Castillón, Margarita Vázquez de Parga y José Ramón Cruz Mundet, junto a dos grandes archiveras, que han sido siempre mis grandes apoyos María Aurelia del Corral y Carmen Sierra, pero en mi desarrollo personal he conocido a dos grandes compañeras de trabajo, María Josefa García Gómez, Subdirectora General del Archivo General de la Administración, que se volcó conmigo en los nuevos caminos que emprendía el AGA y mi madre adoptiva en Vitoria, María José, la agustina de Aragón del Archivo Histórico Provincial de Álava, que supo defender la plaza y la institución archivística en el puesto que le correspondía en las instituciones del País Vasco y que adoptó como a sus hijos a todos los archiveros que tuvimos la suerte de convivir con ella las penalidades de la vida de un archivero en provincias. 
¿Cuáles considera que son las oportunidades y debilidades de dicho archivo?

Empecemos por las oportunidades, lo que en un estudio de avaluación de la calidad entendemos por Fortalezas:

$1^{\circ}$. - El personal, francamente el apoyo, dedicación y amor a su trabajo, que siempre he encontrado entre mis compañeros archiveros, encuadernadores, restauradores, fotógrafos, ordenanzas, mozos, electricistas, calefactores, albañiles, pintores, etc., con algunos mis relaciones de amistad son ya familiares, como si tuviésemos la misma sangre. Si bien en todos los colectivos existen los hijos pródigos y las ovejas negras, pero son las excepciones que confirman la regla, que hace más fuerte la fortaleza del colectivo.

En mis cuarenta años de trabajo en los archivos estatales, tanto en mi etapa en los archivos provinciales y en el AGA, la fortaleza del personal es la cualidad que destaca de la Institución Archivística Española, palabras que escribo en mayúsculas para resaltar su solidez. Cuando trabajas en los archivos tienes siempre la sensación de que has sido olvidado por la institución o administración que lo alberga, que en la distancia aparenta ser un ente administrativo frío y distante, cicatero en cuanto a las retribuciones y gratificaciones, pero que cuando tienen que responder ante una crisis o emergencia nacional o meramente institucional, tanto el ente como el personal, se funden en una fortaleza, con el fin de encarar y solucionar el problema. Siempre analizo los años de la Guerra Civil Española, las décadas del hambre de los años 40 y 50 del siglo $\mathrm{XX}$, la crisis de la transición democrática y recientemente las actuales crisis económicas encadenadas desde el año 2010, agudizada en la actualidad con la actual emergencia sanitaria. En todos los períodos personal e institución se han fusionado como un solo ente y han superado las crisis de los enfrentamientos sociales, políticos, económicos y los más crueles los de las venganzas y las víctimas sin sentido. En estos dos siglos difíciles, es la solidaridad de unos pocos profesionales archiveros lo que ha permitido que los ciudadanos puedan hacer valer sus derechos ante las crisis que amenazan y coartan su identidad, su libertad y su derecho a la verdad.

2.- En segundo lugar, el contenido o los documentos que conservan, estudian y difunden las instituciones archivísticas para garantizar los derechos a la vida, a la libertad, a la propiedad, a la educación, al pensamiento y a la conciencia.

Las instituciones y organismos deben tener claro, que sin los archivos y sin archiveros, sus instituciones y organismos no existirían, como tampoco existiría nuestra propia sociedad, no importa el tipo de soporte del documento, da igual si es real o táctil a la mano o si no conocemos sus componentes etéreos, digitales o electrónicos, pero siempre que se gestionen en una institución archivística, que les dote de identidad y veracidad, podremos dormir tranquilos pensando que nuestra existencia en el universo sigue garantizada.

Si no existiese el contenido documental, sospecho que aceleraríamos el proceso de extinción de la humanidad.

La segunda parte de la pregunta las Debilidades:

Tiene fácil respuesta, lo difícil que es concienciar a la sociedad de la necesidad de conservar los documentos y los archivos.

Desde que el ser humano, plasmó sus sentimientos en expresiones artísticas en piedras, hojas, cortezas, grafitis, pinturas rupestres, ostracas, tablilla de barro cocido, papiros, 
pergaminos, papel, bit electrónicos o cuánticos, la mayor debilidad ha sido su destrucción por la dificultad que entraña la conservación de la memoria del paso de la humanidad por el planeta. Desde las primeras manifestaciones escritas, su mayor debilidad, fue concienciar a la sociedad de la necesidad de guardar y hacer espacio a nuestra cultura.

Desde los tiempos de Aristóteles oímos machaconamente, la frase, "no traigas más papiros, rollos, libros, etc.", hay que tirar las enciclopedias antiguas, ya..., con el móvil”, "hay que ver, ha suspendido y está leyendo una novela", "Yo no he leído un libro en mi vida y no pienso hacerlo", "aquí la rata de archivo" o cuando tu jefe te presenta "como el archivador" a semejanza del perchero. "Te he borrado el disco duro, porque lo necesitaba", "total para qué queremos el archivo si tenemos ordenadores", "Como estos papeles ya no se entienden podemos tirarlos", "Quemamos el archivo y así ganamos espacio...", "Cómo lees en libro en papel o en libro electrónico" y los más ignorantes siempre responden "yo siempre en el libro de toda la vida" ....

Podría escribir una enciclopedia con frases del día a día, escuchadas habitualmente, incluso en nuestros hogares, pero son tan repetitivas que ya no nos damos cuenta de que quizás esa debilidad diaria nos haga perder nuestra identidad, de la que ya no tendremos los medios, los documentos, para recuperarla, la frase lapidaria de ¿para qué guardamos tantos documentos si nadie los va a leer? esa es nuestra mayor debilidad.

2. Hay una frase que, leyendo su obra sobre "Los Archivos del Estado", me hizo comprender la relevancia del momento histórico que estamos viviendo en el ámbito de la gestión documental: “Con la aparición del papel, los documentos y manuscritos que no fueron migrados o copiados al nuevo soporte escriturario pronto dejaron de interesar desapareciendo en el olvido, si no habían sido calificados como tesoros por sus miniaturas, caligrafías o contenidos".

¿Piensa que la documentación que no sea sometida a una transformación digital quedará en el olvido, como sucedió con los manuscritos medievales?

No es una cuestión de pensamiento o creencia, es simplemente lo que la historia nos ha demostrado, hace años Hipólito Escolar Sobrino en su historia del libro nos lo demostraba, hoy Irene Vallejo y su libro "El infinito en un junco", vuelve a resaltar lo mismo.

Podemos reseñar Los grandes hitos históricos de los cambios de soporte:

- El cambio tecnológico del rollo de papiro y de las tabletas de arcilla cocida y de las tabletas de cera, al formato del libro manuscrito en pergamino, provocó la desaparición de los clásicos griegos, de los que solo conservamos aproximadamente un 5 o 10\% de lo que se escribió desde el nacimiento de la escritura. Las culturas orientales y las del norte de África, no fueron descubiertas hasta bien entrado el siglo XIX.

- El cambio tecnológico del manuscrito en pergamino al texto impreso en papel provocó la desaparición del 90\% de los clásicos romanos, la cultura alto medieval y la cultura del Imperio Romano oriental de Constantinopla.

- El cambio tecnológico de la memoria electrónica y la conservación en la nube digital está revolucionando y aniquilando la cultura impresa, la cultura musical e incluso amenaza seriamente a la prensa y con ello a la veracidad de la información.

- Nuestra cultura actual ha presenciado en los siglos XIX y XX, la destrucción de nuestra cultura por motivos religiosos, ideológicos, políticos y los menos justificables los seudocientíficos, como 
las grandes expediciones científicas en busca de culturas antiguas para llenar las vitrinas de los museos, desde las expediciones científicas de los ejércitos napoleónicos y las terribles revoluciones culturales, sociales, políticas y religiosas, son los grandes peligros que amenazan la desaparición de nuestra cultura.

Son precisamente las obras más difundidas las que sobreviven a las catástrofes lo que debemos tener claro es que la implantación de la memoria digital acabará con todo recuerdo de nuestra civilización actual si no migramos nuestros archivos, bibliotecas y museos a los nuevos elementos de difusión digital.

¿Cuáles son los cambios que ha vivido y experimentado en la administración en el ámbito de los archivos en los años de ejercicio profesional?

Cuarenta años es poco tiempo para apreciar los cambios en los archivos administrativos, es tal la acumulación de documentación en papel, que aparentemente seguimos viendo y contemplando los grandes establecimientos archivísticos, sin apreciar en ellos graves alteraciones en su funcionamiento, lo que permite a muchos despreciar la revolución digital, que es imparable.

Si abrimos bien los ojos observamos ya los grandes cambios en la gestión del Estado, están desapareciendo los grandes archivos administrativos en papel de las áreas económicas, fiscales, de gestión de personal y de gestión de los ciudadanos, áreas que mueven el 90\% de la producción documental, es decir que se ha dejado de producir el 90\% de los expedientes en papel que colapsaban los edificios, pero hoy el problema de la Administración es la carencia de memoria electrónica y digital para la conservación de esta gestión a plazos de larga duración, que en términos informáticos es el período de tiempo de conservación de la información antes de suprimirla, para reutilizar el espacio de memoria electrónica y digital. Lo que significa que el plazo de larga conservación en la gestión y conservación del 90\% de la gestión económica y fiscal es de solo cuatro años. Por lo que debemos decidir urgentemente el software y hardware necesario para la conservación permanente de la información que debe conservarse en el Archivo Único de la Administración Electrónica.

¿Considera que los archivos tienen cubiertas sus necesidades de personal y tecnológicas para asumir la transformación digital?

Por supuesto que no. Carecemos de personal y sobre todo de personal altamente cualificado, nuestros responsables archivísticos sigue dando más valor a los conocimientos de idiomas modernos, despreciando los necesarios conocimientos en paleografía, diplomática, las lenguas muertas como el latín, griego, árabe, y se siguen despreciando las nuevas especialidades tecnológicas, acogiéndose a la fórmula negacionista de que queda mucho tiempo para que desaparezca el papel en la administración, quizás lo que debería renovar la administración es la cualificación que necesitan los responsables técnicos que son incapaces de elaborar una planificación archivística en la nueva Era digital.

¿Cuáles considera que son los pasos para seguir en los archivos para tener un papel relevante en este cambio que estamos experimentado?

Son sencillos: 
- Memoria de necesidades

- Elaboración del proyecto de las infraestructuras necesarias

- Modificación de la estructura de personal.

- Plan Estratégico de Implantación del Archivo Único de la Administración Electrónica y Digital.

3. Con su habilidad para sacar monografías de gran interés y calidad en tiempo récord en el ámbito de la investigación histórica

¿Qué motivo le hizo optar por los archivos y no por la investigación histórica en su elección profesional?

Muchas gracias por el piropo sobre mi habilidad en sacar monografías, porque no sé si me leen, sé que ciertos sectores de asociaciones de archiveros y responsables administrativos no están de acuerdo con mis planteamientos, lo que me parece muy bien, porque yo no le encuentro sentido a las asociaciones profesionales, ya no pertenezco a ninguna, es notorio y conocido que no comparto ciertos criterios profesionales.

Respecto a mis motivaciones, me hubiese encantado ser pintor y arqueólogo y vivir las mieles de la bohemia, pero no sus estrecheces económicas. Mi madre hubiese querido que hiciese medicina y seguir los pasos del primo rojo de mi abuela materna, Severo Ochoa, que estuvo a punto también de ser mi abuelo materno, pero sus padres impidieron el noviazgo de dos primos hermanos, Severo fue a estudiar a la Universidad de Sevilla, y mi abuela en Segovia se enamoró de un cadete de artillería, mi abuelo paterno D. José Oliveda Medrano, descendiente de la guerrera estirpe de la nobleza catalana de Mollet de Perelada, en las tierras vinícolas gerundenses de la antesala del Pirineo, hombre que se hizo a sí mismo y educó a sus hermanos, quienes quedaron huérfanos en la infancia, por la epidemia de gripe de 1919, hombre de estirpe de nobleza catalana, fiel a su Rey en los tiempos convulsos de siglo XX.

La verdad es que la historia y los archivos me han proporcionado el desarrollo intelectual que necesitaba.

Respecto a la investigación, he desarrollado esta faceta en el puro aspecto de la archivística, no he escrito manuales técnicos, porque mi experiencia en la enseñanza, enseguida me hizo ver el gran período de transformación que estaba sufriendo nuestra profesión, por lo que mis textos y mis planificaciones de futuro se quedaron en las memorias y planificaciones en los centros archivísticos en los que he desarrollado mi trabajo.

¿Piensa que los archiveros deben de contribuir a la historiografía, ejerciendo no sólo como documentalistas sino también escribiendo sobre los textos que catalogan?

Entiendo que nuestra profesión es el punto de partida de lo que hace unos años empezó a denominarse historiografía. Durante mi período de formación, en la escuela de biblioteconomía y documentación, aprendí sobre todo a planificar según los principios de la organización y método, lo que ahora conocemos como "Calidad", entonces comprendí que para describir un fondo documental había que establecer un método de trabajo consistente en:

- Investigación de la Institución: historia, legislación, organigrama, series documentales que produce

- Establecimiento del Cuadro de Clasificación 
- Relación del inventario, somero, analítico...

- Disposición física de conservación de la documentación: clasificación y ordenación física si por su volumen es viable; clasificación y ordenación intelectual cuando no puede realizarse físicamente, etc.

- Memoria final del tratamiento realizado sobre la organización del Fondo Documental.

¿Cuáles consideraría que han sido sus mayores descubrimientos entorno a la figura de Miguel de Cervantes y Saavedra?

Tengo que aclarar, que cuando me enfrenté a D. Miguel de Cervantes Saavedra, en el año 2014, yo solo iba a preparar un pequeño folleto para una exposición del fondo documental del Archivo del Corregimiento de Alcalá de Henares del siglo XV al siglo XIX y de los fondos judiciales del mismo y su integración en el Juzgado de Primera Instancia, creado en el siglo XIX.

Dicha exposición nunca se hizo, pero mi estudio previo sobre Cervantes me hizo constatar los siguientes hechos:

- Que la única biografía oficial había sido escrita por Mayans y Siscar en el siglo XVIII, basándose únicamente en los propios escritos literarios y novelescos escritos por el propio Cervantes.

- Qué desde entonces, los archivos han ido presentando la documentación original sobre Cervantes, pero siempre los documentos debían acomodarse a la calificación que había establecido sobre él Mayans.

- Qué los documentos que contradicen esta versión son despreciados, o se esconden, se destruyen o se hacen desaparecer para no acabar con el mito del Cervantes pobre al que mantienen sus hermanas.

- Que los facultativos del Cuerpo de Archiveros, Bibliotecarios y Arqueólogos son los que más habían dado a conocer a Cervantes a la historia, pero que sus aportaciones eran despreciadas si contradecían lo oficialmente admitido por las cátedras y academias literarias.

- En los volúmenes de los Apuntes para una biografía de Miguel de Cervantes, se dan a conocer las casuísticas históricas, académicas, literarias y militares, de este hombre, contemporáneo de Shakespeare, que abren nuevas perspectivas a los futuros estudios sobre Cervantes, hombre totalmente desconocido, pero al que debemos dotar de su identidad dentro de nuestro trabajo archivístico.

¿Deberíamos replantearnos nuestra visión de Miguel de Cervantes teniendo en cuenta dichos descubrimientos?

No soy yo el que debe contestar esta pregunta.

4. En último lugar, y en su calidad de gran conocedor de la archivística, nos gustaría que nos diera algún consejo profesional para afrontar los tiempos que corren y un consejo para los jóvenes historiadores que para que se animen a visitar las instituciones archivísticas.

Cuando se acerquen a una institución archivística, deben ser francos y explicar lo que buscan, solo así el archivero podrá asesorarle sobre los archivos y documentos que necesita para su 
investigación y que las instituciones deben permitir el libre acceso digital a los archivos y documentos históricos.

\section{Referencias}

Alfonso Dávila Oliveda. (1997). La acción de las Juntas Generales alavesas reflejadas en los protocolos notariales. En Comunicaciones libres: Congreso Internacional sobre Sistemas de Información Histórica, 6, 7 y 8 de noviembre de 1997, Vitoria-Gasteiz / coord. por Manuel Balado Ruiz-Gallegos, José Antonio García Regueiro, María José de la Fuente y de la Calle, Vol. 2 (363-366). Juntas Generales de Álava = Arabako biltzar nagusiak: Vitoria-Gasteiz.

Alfonso Dávila Oliveda. (2010). Los archivos del Estado: qué son y cómo se tratan. Trea.

Alfonso Dávila Oliveda. (2015). Miguel de Cervantes: Apuntes para una biografía. Vol. 1. Soldado poeta (1547-1585). Círculo Rojo.

Alfonso Dávila Oliveda. (2016). Miguel de Cervantes: Apuntes para una biografía. Vol. 2. El agente del Rey, predestinado para el teatro, que se dedicaba a los negocios. (1586-1595). Círculo Rojo.

Alfonso Dávila Oliveda. (2016). Las universidades de Alcalá y México en el siglo XVI el patrocinio de la monarquía y la familia Cervantes. En Historia universitaria de España y América / José Manuel Calderón Ortega (aut.), Manuel Casado Arboniés (aut.), Alejandro Ramón Díez Torre (aut.) (447-484). Alcalá de Henares: Universidad de Alcalá de Henares.

Alfonso Dávila Oliveda. (2017). Miguel de Cervantes: Apuntes para una biografía. Vol. 3.1. Que Acabó con Hasan Baja, Virrey de Trípoli y Argel (1595-1601). Círculo Rojo.

Alfonso Dávila Oliveda. (2017). La Revolución Universitaria Cisneriana Sobre el Modelo Universitario Medieval. Amazon Mexico Services, Inc.

Alfonso Dávila Oliveda. (2017). El patrocinio de Juana de Austria sobre el Real Colegio-Convento de agustinos calzados de San Agustín de Alcalá de Henares. En Fundadores y patronos universitarios, Alcalá de Henares, siglo XVI: colegios cisnerianos, colegios-convento y colegios seculares / Manuel Casado Arboniés (ed. lit.), Carmen Román Pastor (ed. lit.) (149172). Alcalá de Henares: Universidad de Alcalá de Henares.

Alfonso Dávila Oliveda. (2018). Panamá. La Castilla del Oro: Pedrarias Dávila. El gran justador de Castilla. Amazon Mexico Services, Inc.

Alfonso Dávila Oliveda. (2019). Miguel de Cervantes: Apuntes para una biografía. Vol. 3. El espía (1595-1603). Círculo Rojo.

Alfonso Dávila Oliveda. (2019). Miguel de Cervantes y Alcalá de Henares. Guía de Itinerario. Círculo Rojo.

Alfonso Dávila Oliveda. (2019). La Universidad Autónoma de México y los familiares de Miguel de Cervantes. Amazon Mexico Services, Inc.

Alfonso Dávila Oliveda. (2019). Archivo General Central Del Reino de España. Los documentos desaparecidos el 9 de agosto de 1939. En De Palacio a Casa de los Arqueólogos: pasado y futuro del Palacio Arzobispal de Alcalá de Henares. coord. por María Carrillo (93-107). Alcalá de Henares: Comunidad de Madrid, Museo Arqueológico Regional. 


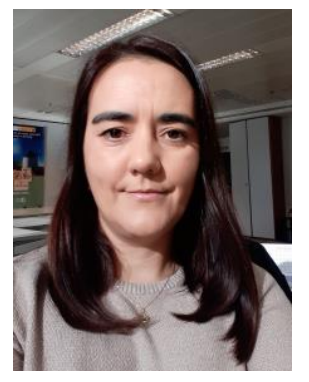

Ana Naseiro Ramudo

Archivera del Cuerpo Facultativo de Archivos

Licenciada en Historia por la Universidad de Santiago de Compostela, ejerce Facultativa de Archivos de la Administración General del Estado, ha trabajado en el Archivo General de la Administración del Estado como jefa de descripción y jefa de valoración y acceso. Y actualmente es la Jefa del Archivo de la Oficina Española de Patentes y Marcas. Forma parte de la Junta Directiva de SEDIC (Sociedad Española de Documentación e Información Científica), desde el año 2019, donde ejerce como vocal. E-mail: anaramudonase@gmail.com

- $\quad$ https://es.linkedin.com/in/ana-naseiro-ramudo-8867b676

- https://es-es.facebook.com/ana.naseiroramudo 